\title{
Motor Side-View Recognition System Based on Wavelet Entropy and Naïve Bayesian Classifier
}

\author{
Yiyang Chen ${ }^{1, *}$ and Matben Suchkov ${ }^{2}$ \\ ${ }^{1}$ Jiangsu Key Laboratory of 3D Printing Equipment and Manufacturing, Nanjing, Jiangsu 210042, China \\ ${ }^{2}$ Institute of Management, Economics and Finance, Kazan Federal University, Kazan, 420008, Russia \\ ${ }^{*}$ Corresponding author
}

\begin{abstract}
As the traffic accident becomes a serious problem, we need some more efficient methods to identify the car. Luckily, we can use artificial intelligence to recognize the motors by using side-view images. It will help a lot to solve the traffic accident. We used the wavelet entropy to extract the feature of the images. Then we employed the naïve Bayesian theory as the classifier. And we used 10-fold cross validation in our experiment. We used a three-level decomposition for WE. It got an overall accuracy of $75 \%$ in recognizing motors. In the future we will try to improve the accuracy of this method and try to identify cars form different brand.
\end{abstract}

Keywords-artificial intelligence; wavelet entropy; naïve Bayesian classifier; cross validation

\section{INTRODUCTION}

As the motors become more and more popular in china, the traffic accident become more serious and frequent than before. In these situations, we need to recognize the motors. However, it is difficult for us to extract the pictures of the front of the motors from the camera [1]. But it is easier for us to get the images of the side view of the motors. This technology will help police to solve the accident and judge the responsibility of the motors involved in the accident.

At the present, there is not a technology can recognize the car brand from the side view. We searched on Web of Science, Engineering Index, Scopus, IEEE, and other well-known databases, we did not find some related articles, so we believe that we are one of the first team to work on it.

We use Wavelet Entropy (WE) [2-5] to obtain the features of the motor images. This method is better than the Scale Invariant Feature Transform (SIFT) [6-8], which will cause some features match problems. WE has a lot advantages, it can isolate each images which will make the recognition easier. And also WE had some benefits over Fourier transforms on specific frequencies.

The naïve Bayesian (NB) classifier $[9,10]$ is used to identify the motors brand from 20 images. We assume every feature the WE extract is independent of any other feature, and likelihood of each feature is same. This classifier can be set very efficiently and only need a small number of data to estimate the parameters, which is more appropriate than other classifier in this survey.

\section{METHODS}

\section{A. Wavelet Entropy}

Our method is made up by four stages, including shooting the images of motors, image processing, obtaining features of motors, and NB classification.

Our method is to us e wavelet entropy (WE) to get the features of motors. WE is based on wavelet entropy and information entropy [11]. The formula can be described like this:

$$
\mathrm{W}_{\psi}(a, c)=\int_{-\infty}^{+\infty} x(t) \psi_{a, c}(t) d t
$$

And

$$
\mathrm{W}_{a, c}(t)=\frac{1}{\sqrt{|a|}} \psi\left(\frac{t-c}{a}\right), a \neq 0, c \in R
$$

In the formula, a means scale factor, c means translation factor. $\Psi$ is called orthonormal wavelet, which is decided by factor a and c. And the formula above is called continuous wavelet transform (CWT). However, if the samples of the wavelets are discrete, the transform will become discrete wavelet transform (DWT), which is used in our method. DWT has an advantage which can get frequency and location information at the same time [12-18].

We will use two-dimensional (2D) DWT twice. After the first DWT, we can get four images, the upper-left is the lowpass filtered images, which contain the most features of the image [19-27]. And the use 2D-DWT to the upper-left image again, and we get seven images, each including different features. The low frequency images contain the most information, while the upper-right contain the information about horizontal features, the left lower contain the information about vertical feature, and the right lower contain the information about diagonal features [28-35].

As it shown in Figure $I, x[n]$ is input, $g[n]$ means low pass filter, $\mathrm{h}[\mathrm{n}]$ means high pass filter, while $\mathrm{Q}$ means a 
downsampling filter, and we take the factor of 2 as an example. The Figure II demonstrate the twice 2D-DWT.

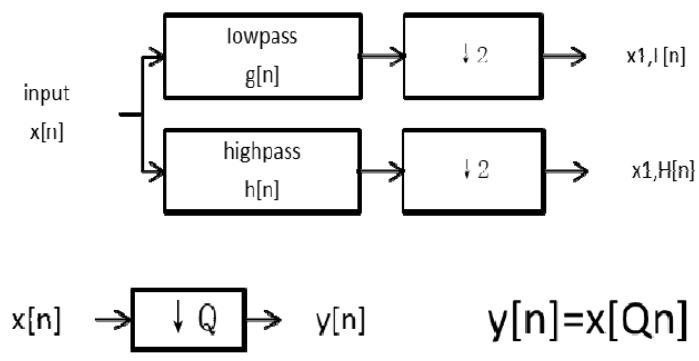

FIGURE I. 2D-DWT

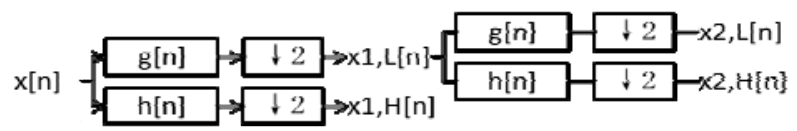

FIGURE II. TWICE 2D-DWT

\section{B. Nä̈ve Bayesian Classifier}

In our method, naïve Bayesian (NB) classifier is used. Bayes theory is an important tool for dealing with uncertain information. As an uncertain method for reasoning, it depends on the theory of probability and statistics and it has a solid mathematical basis [36]. NB classifier is a learning method with supervision. We assume that each value of feature is independent of other features, which make the classifier easier and efficient, but also accurate. So, it will save the computing time and memory space of the computer. Naïve Bayesian classifier can be described as following formula [37]:

$$
\mathrm{P}(B \mid A)=\frac{\mathrm{P}(B \mid A) \mathrm{P}(B)}{\mathrm{P}(A)}
$$

And if we can get the value of $\mathrm{P}(\mathrm{A} \mid \mathrm{B}), \mathrm{P}(\mathrm{B}), \mathrm{P}(\mathrm{A})$, then we can find the value of $\mathrm{P}(\mathrm{B} \mid \mathrm{A})$.

Now we make this formula easier to understand.

$$
\mathrm{P}(\text { outcomes } \mid \text { features })=\frac{\mathrm{P}(\text { features } \mid \text { outcomes }) \mathrm{P}(\text { outcomes })}{\mathrm{P}(\text { features })}
$$

In the formula, sometimes there are different features.

$\mathrm{P}($ outcomes $\mid$ features $)=\mathrm{P}\left(\right.$ outcomes features ${ }_{1}$, features $_{2}$, features $\left._{3} \ldots\right)$

Now we need some data to set our classifier. We need to teach our NB classifier to how to identify the brand of motors. Now we need a database, and we calculate the likelihood of each features. Then we have the value of the likelihood of each feature.

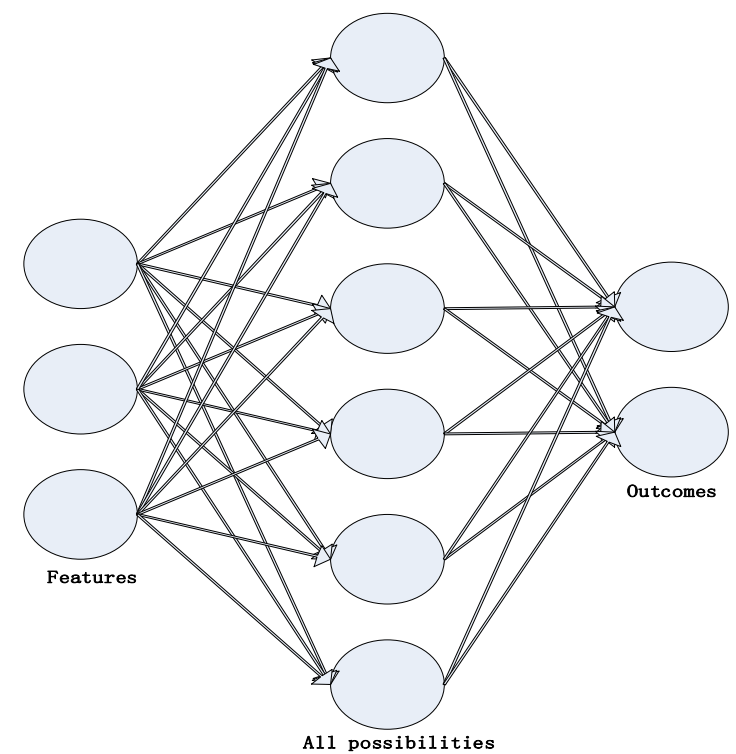

FIGURE III. NB CLASSIFIER

We assume that every feature is independent. So we have:

$$
\begin{aligned}
& \mathrm{P}(\text { features } \mid \text { outcomes })=\mathrm{P}\left(\text { features }_{1} \mid \text { outcomes }\right) \\
& * \mathrm{P}\left(\text { features }_{2} \mid \text { outcomes }^{*}\right) \mathrm{P}\left(\text { features }_{3} \mid \text { outcomes }\right) \\
& =\prod_{x=1}^{n} \mathrm{P}\left(\text { features }_{x} \mid \text { outcomes }\right)
\end{aligned}
$$

From Figure III, we can know how NB classifier works. We calculate the likelihood of each possibility and choose the most likely situation to be outcomes.

After 2D DWT, we can extract the features of the images. Now we know the likelihood of each features, so we can solve the problems by using naïve Bayesian classifier.

\section{EXPERIMENTS AND RESULTS}

The dataset is shown below in Figure IV. Each image was resized to $255 \times 255$. We have in total 80 Ford vehicle images and 80 non-Ford vehicle images, including Buick, Hyundai, Shanghai Volks, and Toyota.

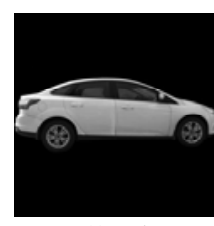

(a) Ford

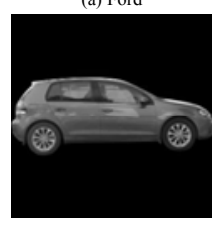

(d) Shanghai volks

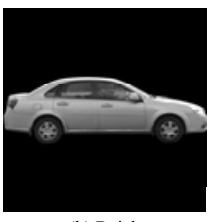

(b) Buick

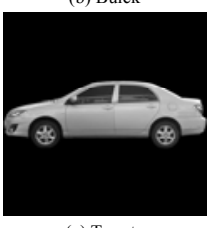

(e) Toyota

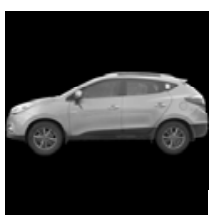

(c) Hyundai
FIGURE IV. SAMPLES OF DATASET 


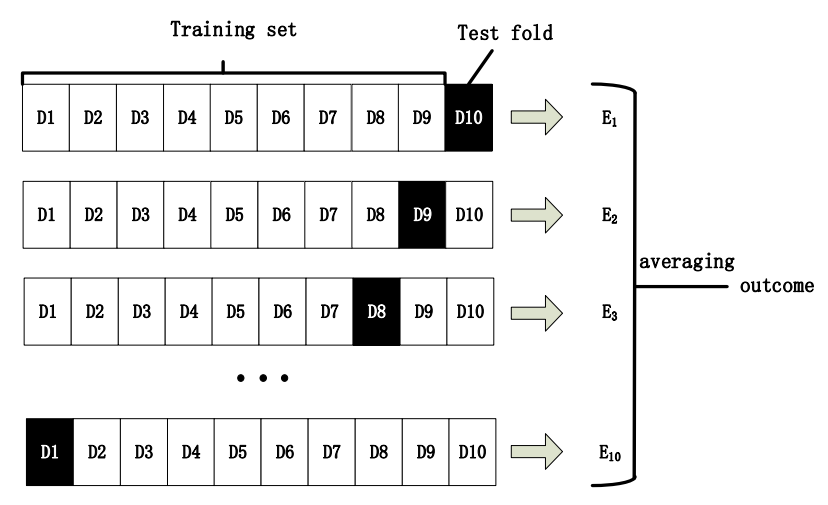

FIGURE V. ILLUSTRATION OF 10-FOLD CROSS VALIDATION

We used a three-level decomposition for WE. The statistical results of $10 \times 10$-fold cross validation were shown below in Table I, Table II, and Table III, respectively. The average sensitivity is $75.25 \pm 2.93 \%$, the average specificity is $75.25 \pm 3.27 \%$, and the average accuracy is $75.25 \pm 0.67 \%$.

\section{TABLE I. SENSITIVITY OF OUR METHOD BY A 10X10-FOLD CROSS} VALIDATION

\begin{tabular}{|l|l|l|l|l|l|l|l|l|l|l|l|}
\hline Sen & F1 & F2 & F3 & F4 & F5 & F6 & F7 & F8 & F9 & F10 & Total \\
\hline R1 & 100.0 & 62.5 & 100.0 & 87.5 & 100.0 & 75.0 & 62.5 & 50.0 & 87.5 & 37.5 & 76.25 \\
\hline R2 & 87.5 & 87.5 & 75.0 & 75.0 & 87.5 & 75.0 & 75.0 & 75.0 & 62.5 & 50.0 & 75.00 \\
\hline R3 & 100.0 & 87.5 & 75.0 & 50.0 & 75.0 & 75.0 & 87.5 & 62.5 & 62.5 & 100.0 & 77.50 \\
\hline R4 & 87.5 & 87.5 & 75.0 & 62.5 & 62.5 & 75.0 & 75.0 & 100.0 & 62.5 & 100.0 & 78.75 \\
\hline R5 & 100.0 & 87.5 & 75.0 & 75.0 & 100.0 & 75.0 & 50.0 & 100.0 & 75.0 & 62.5 & 80.00 \\
\hline R6 & 75.0 & 62.5 & 75.0 & 75.0 & 75.0 & 62.5 & 62.5 & 75.0 & 87.5 & 87.5 & 73.75 \\
\hline R7 & 75.0 & 62.5 & 75.0 & 87.5 & 75.0 & 75.0 & 87.5 & 62.5 & 75.0 & 62.5 & 73.75 \\
\hline R8 & 62.5 & 87.5 & 50.0 & 87.5 & 87.5 & 75.0 & 75.0 & 62.5 & 75.0 & 87.5 & 75.00 \\
\hline R9 & 75.0 & 87.5 & 62.5 & 75.0 & 62.5 & 87.5 & 50.0 & 75.0 & 75.0 & 62.5 & 71.25 \\
\hline R10 & 50.0 & 75.0 & 87.5 & 87.5 & 75.0 & 62.5 & 75.0 & 37.5 & 75.0 & 87.5 & 71.25 \\
\hline
\end{tabular}

TABLE II. SPECIFICITY OF OUR METHOD BY A 10X10-FOLD CROSS VALIDATION

\begin{tabular}{|l|l|l|l|l|l|l|l|l|l|l|l|}
\hline Spc & F1 & F2 & F3 & F4 & F5 & F6 & F7 & F8 & F9 & F10 & Total \\
\hline R1 & 75.0 & 62.5 & 75.0 & 75.0 & 37.5 & 100.0 & 87.5 & 62.5 & 87.5 & 62.5 & 72.50 \\
\hline R2 & 62.5 & 75.0 & 62.5 & 75.0 & 75.0 & 87.5 & 100.0 & 75.0 & 87.5 & 75.0 & 77.50 \\
\hline R3 & 75.0 & 75.0 & 50.0 & 62.5 & 75.0 & 87.5 & 87.5 & 62.5 & 75.0 & 75.0 & 72.50 \\
\hline R4 & 62.5 & 37.5 & 62.5 & 75.0 & 75.0 & 75.0 & 100.0 & 75.0 & 62.5 & 75.0 & 70.00 \\
\hline R5 & 50.0 & 75.0 & 75.0 & 100.0 & 62.5 & 87.5 & 75.0 & 50.0 & 87.5 & 62.5 & 72.50 \\
\hline R6 & 75.0 & 50.0 & 87.5 & 75.0 & 75.0 & 87.5 & 87.5 & 62.5 & 87.5 & 75.0 & 76.25 \\
\hline R7 & 75.0 & 75.0 & 62.5 & 87.5 & 75.0 & 75.0 & 62.5 & 75.0 & 87.5 & 100.0 & 77.50 \\
\hline R8 & 87.5 & 75.0 & 87.5 & 62.5 & 75.0 & 87.5 & 62.5 & 87.5 & 50.0 & 75.0 & 75.00 \\
\hline R9 & 87.5 & 87.5 & 75.0 & 62.5 & 100.0 & 100.0 & 62.5 & 62.5 & 87.5 & 62.5 & 78.75 \\
\hline R10 & 75.0 & 50.0 & 87.5 & 87.5 & 75.0 & 75.0 & 50.0 & 100.0 & 100.0 & 100.0 & 80.00 \\
\hline
\end{tabular}

TABLE III. ACCURACY OF OUR METHOD BY A 10X10-FOLD CROSS VALIDATION

\begin{tabular}{|l|l|l|l|l|l|l|l|l|l|l|l|}
\hline Acc & F1 & F2 & F3 & F4 & F5 & F6 & F7 & F8 & F9 & F10 & Total \\
\hline R1 & 87.50 & 62.50 & 87.50 & 81.25 & 68.75 & 87.50 & 75.00 & 56.25 & 87.50 & 50.00 & 74.38 \\
\hline R2 & 75.00 & 81.25 & 68.75 & 75.00 & 81.25 & 81.25 & 87.50 & 75.00 & 75.00 & 62.50 & 76.25 \\
\hline R3 & 87.50 & 81.25 & 62.50 & 56.25 & 75.00 & 81.25 & 87.50 & 62.50 & 68.75 & 87.50 & 75.00 \\
\hline R4 & 75.00 & 62.50 & 68.75 & 68.75 & 68.75 & 75.00 & 87.50 & 87.50 & 62.50 & 87.50 & 74.38 \\
\hline R5 & 75.00 & 81.25 & 75.00 & 87.50 & 81.25 & 81.25 & 62.50 & 75.00 & 81.25 & 62.50 & 76.25 \\
\hline R6 & 75.00 & 56.25 & 81.25 & 75.00 & 75.00 & 75.00 & 75.00 & 68.75 & 87.50 & 81.25 & 75.00 \\
\hline R7 & 75.00 & 68.75 & 68.75 & 87.50 & 75.00 & 75.00 & 75.00 & 68.75 & 81.25 & 81.25 & 75.63 \\
\hline R8 & 75.00 & 81.25 & 68.75 & 75.00 & 81.25 & 81.25 & 68.75 & 75.00 & 62.50 & 81.25 & 75.00 \\
\hline R9 & 81.25 & 87.50 & 68.75 & 68.75 & 81.25 & 93.75 & 56.25 & 68.75 & 81.25 & 62.50 & 75.00 \\
\hline R10 & 62.50 & 62.50 & 87.50 & 87.50 & 75.00 & 68.75 & 62.50 & 68.75 & 87.50 & 93.75 & 75.63 \\
\hline
\end{tabular}

The box plot of proposed WE+NB method is shown in Figure VI. We can observe the accuracy has a slight variation compared to sensitivity and specificity.

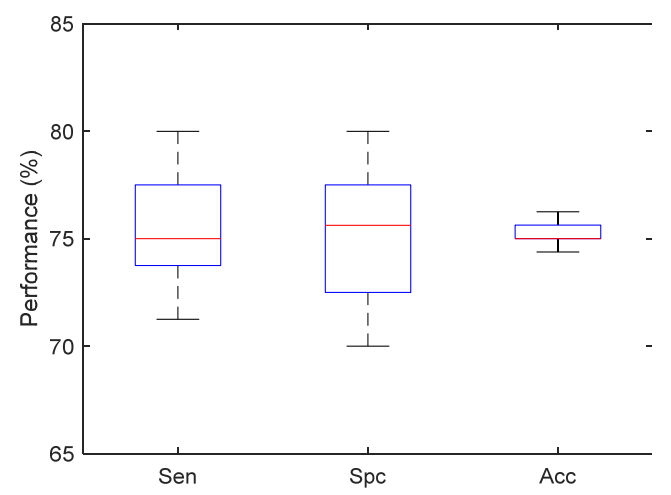

FIGURE VI. BOX PLOT OF PERFORMANCES OF PROPOSED METHOD

Finally, we compared the results of using different decomposition levels of wavelet entropy. The results are listed in Table IV. We can observe that 3-level decomposition obtained the greatest performance. While 1-level decomposition yields a sensitivity of $68.50 \pm 3.81 \%$, a specificity of $68.00 \pm 3.34 \%$, and an accuracy of $68.25 \pm 1.21 \%$. The 2-level decomposition yields a sensitivity of $73.25 \pm 2.90 \%$, a specificity of $73.50 \pm 3.81 \%$, and an accuracy of $73.38 \pm$ $0.73 \%$. The 4-level decomposition yields a sensitivity of $74.13 \pm 2.89 \%$, a specificity of $74.13 \pm 2.70 \%$, and an accuracy of $74.13 \pm 0.53 \%$. In the future, some optimization techniques (such as bacterial chemotaxis optimization [38, 39], biogeography-based optimization, multiverse optimizer, etc.) shall be tested to check whether they can improve the classification performance.

TABLE IV. COMPARISON OF DIFFERENT DECOMPOSITION LEVELS

\begin{tabular}{|c|c|c|c|}
\hline Decomposition & Sensitivity & Specificity & Accuracy \\
\hline 1-level & $68.50 \pm 3.81$ & $68.00 \pm 3.34$ & $68.25 \pm 1.21$ \\
\hline 2-level & $73.25 \pm 2.90$ & $73.50 \pm 3.81$ & $73.38 \pm 0.73$ \\
\hline $\begin{array}{c}\text { 3-level } \\
\text { (Proposed) }\end{array}$ & $75.25 \pm 2.93$ & $75.25 \pm 3.27$ & $75.25 \pm 0.67$ \\
\hline 4-level & $74.13 \pm 2.89$ & $74.13 \pm 2.70$ & $74.13 \pm 0.53$ \\
\hline
\end{tabular}




\section{CONCLUSIONS}

From the experiment, we can find that our method worked, and it can be used to identify the motors. But in our experiment, we just took the Ford brand as the examples, which achieved the accuracy of $75 \%$. But the accuracy is not high when test different brands. Hence, our next step is to make some improvements in identifying different brands and we are trying to achieve a high accuracy in detecting. And in our experiment, the dataset is small, which just contains 160 images. We will enlarge it in our next step.

\section{REFERENCES}

[1] Park, H., et al., Automatic Identification of Driver's Smartphone Exploiting Common Vehicle-Riding Actions. IEEE Transactions on Mobile Computing, 2018. 17(2): p. 265-278

[2] Atangana, A., Application of stationary wavelet entropy in pathological brain detection. Multimedia Tools and Applications, 2018. 77(3): p. 3701-3714

[3] Wu, X., Tea category identification based on optimal wavelet entropy and weighted $k$-Nearest Neighbors algorithm. Multimedia Tools and Applications, 2018. 77(3): p. 3745-3759

[4] Yang, M. Leaf recognition for plant classification based on wavelet entropy and back propagation neural network. in 10th International Conference on Intelligent Robotics and Applications (ICIRA). 2017. Guilin, China: Springer. p. 367-376

[5] Jia, W.-J. and E. Lee. Ford Motor Side-View Recognition System Based on Wavelet Entropy and Back Propagation Neural Network and Levenberg-Marquardt Algorithm. in Eighth International Symposium on Parallel Architectures, Algorithms and Programming (PAAP). 2017. Haikou, China: Springer Singapore. p. 3-12

[6] Pan, H., C. Zhang, and Y. Tian, RGB-D image-based detection of stairs, pedestrian crosswalks and traffic signs. Journal of Visual Communication and Image Representation, 2014. 25(2): p. 263-272

[7] Tian, Y.L. Camera-Based Signage Detection and Recognition for Blind Persons. in 13th International Conference on Computers Helping People with Special Needs (ICCHP). 2012. Linz, AUSTRIA: Springer-Verlag Berlin. p. 17-24

[8] Tian, Y. Detecting stairs and pedestrian crosswalks for the blind by RGBD camera. in IEEE International Conference on Bioinformatics and Biomedicine Workshops (BIBMW). 2012. Philadelphia, PA, USA: IEEE. p. $732-739$

[9] Ontivero-Ortega, M., et al., Fast Gaussian Naïve Bayes for searchlight classification analysis. NeuroImage, 2017. 163: p. 471-479

[10] Zhou, X. Detection of pathological brain in MRI scanning based on wavelet-entropy and naive Bayes classifier. in International Conference on Bioinformatics and Biomedical Engineering (IWBBIO). 2015. Granada, Spain: Springer International Publishing. p. 201-209

[11] Sehgal, V., et al., Application of multi-scale wavelet entropy and multiresolution Volterra models for climatic downscaling. Journal of Hydrology, 2018. 556: p. 1078-1095

[12] Liu, G., Pathological brain detection in MRI scanning by wavelet packet Tsallis entropy and fuzzy support vector machine. SpringerPlus, 2015. 4(1): Article ID. 716

[13] Yang, J.Q., et al., A Novel Compressed Sensing Method for Magnetic Resonance Imaging: Exponential Wavelet Iterative ShrinkageThresholding Algorithm with Random Shift. International Journal Of Biomedical Imaging, 2016: Article ID. 9416435

[14] Sun, P., Preliminary research on abnormal brain detection by waveletenergy and quantum-behaved PSO. Technology and Health Care, 2016. 24(s2): p. S641-S649

[15] Yang, M., Dual-Tree Complex Wavelet Transform and Twin Support Vector Machine for Pathological Brain Detection. Applied Sciences, 2016. 6(6): Article ID. 169

[16] Li, Y., Detection of Dendritic Spines using Wavelet Packet Entropy and Fuzzy Support Vector Machine. CNS \& Neurological Disorders - Drug
Targets, 2017. 16(2): p. 116-121

[17] Li, P. and G. Liu, Pathological Brain Detection via Wavelet Packet Tsallis Entropy and Real-Coded Biogeography-based Optimization. Fundamenta Informaticae, 2017. 151(1-4): p. 275-291

[18] Rao, R.V., Abnormal Breast Detection in Mammogram Images by Feedforward Neural Network trained by Jaya Algorithm. Fundamenta Informaticae, 2017. 151: p. 191-211

[19] Phillips, P., Intelligent facial emotion recognition based on stationary wavelet entropy and Jaya algorithm. Neurocomputing, 2018. 272: p. 668-676

[20] Gorriz, J.M., Multivariate approach for Alzheimer's disease detection using stationary wavelet entropy and predator-prey particle swarm optimization. Journal of Alzheimer's Disease, 2017, DOI: 10.3233/JAD170069.

[21] Nayak, D.R., Detection of unilateral hearing loss by Stationary Wavelet Entropy. CNS \& Neurological Disorders - Drug Targets, 2017. 16(2): p. $15-24$

[22] Lu, H.M., Facial Emotion Recognition Based on Biorthogonal Wavelet Entropy, Fuzzy Support Vector Machine, and Stratified Cross Validation. IEEE Access, 2016. 4: p. 8375-8385

[23] Gorriz, J.M. and J. Ramírez, Wavelet entropy and directed acyclic graph support vector machine for detection of patients with unilateral hearing loss in MRI scanning. Frontiers in Computational Neuroscience, 2016. 10: Article ID. 160

[24] Zhou, X.-X., Comparison of machine learning methods for stationary wavelet entropy-based multiple sclerosis detection: decision tree, $k$ nearest neighbors, and support vector machine. Simulation, 2016. 92(9): p. 861-871

[25] Zhou, X.X. and G.S. Zhang, Detection of abnormal MR brains based on wavelet entropy and feature selection. IEEJ Transactions on Electrical and Electronic Engineering, 2016. 11(3): p. 364-373

[26] Sun, P., Pathological brain detection based on wavelet entropy and $\mathrm{Hu}$ moment invariants. Bio-Medical Materials and Engineering, 2015. 26(s1): p. 1283-1290

[27] Phillips, P., Z. Dong, and J. Yang, Pathological brain detection in magnetic resonance imaging scanning by wavelet entropy and hybridization of biogeography-based optimization and particle swarm optimization. Progress In Electromagnetics Research, 2015. 152: p. 4158

[28] Huo, Y., Feature Extraction of Brain MRI by Stationary Wavelet Transform and its Applications. Journal of Biological Systems, 2010. 18(S): p. 115-132

[29] Zhang, Y.D., et al., An improved reconstruction method for CS-MRI based on exponential wavelet transform and iterative shrinkage/thresholding algorithm. Journal of Electromagnetic Waves and Applications, 2014. 28(18): p. 2327-2338

[30] Yu, D., et al., Exponential wavelet iterative shrinkage thresholding algorithm with random shift for compressed sensing magnetic resonance imaging. IEEJ Transactions on Electrical and Electronic Engineering, 2015. 10(1): p. 116-117

[31] Yang, J., Preclinical diagnosis of magnetic resonance (MR) brain images via discrete wavelet packet transform with Tsallis entropy and generalized eigenvalue proximal support vector machine (GEPSVM). Entropy, 2015. 17(4): p. 1795-1813

[32] Liu, A., Magnetic resonance brain image classification via stationary wavelet transform and generalized eigenvalue proximal support vector machine. Journal of Medical Imaging and Health Informatics, 2015. 5(7): p. $1395-1403$

[33] Dong, Z., et al., Exponential wavelet iterative shrinkage thresholding algorithm for compressed sensing magnetic resonance imaging. Information Sciences, 2015. 322: p. 115-132

[34] Yang, J., Identification of green, Oolong and black teas in China via wavelet packet entropy and fuzzy support vector machine. Entropy, 2015. 17(10): p. 6663-6682

[35] Chen, M., Y. Li, and L. Han, Detection of dendritic spines using waveletbased conditional symmetric analysis and regularized morphological shared-weight neural networks. Computational and Mathematical Methods in Medicine, 2015: Article ID. 454076 
[36] Sharma, M. and M. Bilgic, Learning with rationales for document classification. Machine Learning, 2018. 107(5): p. 797-824

[37] UI Haq, M.A., et al. Indoor Localization Using Improved Multinomial Naïve Bayes Technique. in Proceedings of the Third International AfroEuropean Conference for Industrial Advancement - AECIA 2016. 2018. Cham: Springer International Publishing. p. 321-329

[38] $\mathrm{Wu}, \mathrm{L}$., Weights optimization of neural network via improved BCO approach. Progress in Electromagnetics Research, 2008. 83: p. 185-198

[39] Zhang, Y., Stock market prediction of S\&P 500 via combination of improved BCO approach and BP neural network. Expert systems with applications, 2009. 36(5): p. 8849-8854 